\title{
LA MUJER DIRECTIVA. LA PRESENCIA DE LA MUJER EN LOS CONSEJOS DE ADMINISTRACIÓN DE LAS COMPAÑÍAS DEL IBEX 35
}

\section{WOMEN IN MANAGEMENT. THE PRESENCE OF WOMEN ON THE BOARDS OF DIRECTORS OF IBEX 35 COMPANIES}

Jose María Biedma Ferrer

Universidad de Cádiz

\section{RESUMEN}

La presencia de las mujeres en los puestos directivos ha mejorado con el paso del tiempo. No obstante, como demuestra la literatura, la representación de la mujer en puestos de responsabilidad no es suficiente, a pesar de los efectos beneficiosos que la misma podría tener. En este trabajo se estudian diversas cuestiones, tales como los beneficios que puede generar la incorporación de la mujer a las organizaciones, así como las principales barreras existentes para acceder a puestos directivos - de responsabilidad. También se aborda la evolución de la presencia de la mujer en los consejos de administración de las empresas del IBEX 35 en el período de 2009-2013, y la tipología de las consejeras de dichas empresas. Los resultados del presente estudio vienen a confirmar que, si bien la presencia de la mujer en los consejos de las empresas cotizadas se ha incrementado, aún es insuficiente, quedando por debajo del porcentaje recomendado en la norma. Con respecto a la tipología de las consejeras de dichas empresas, y de acuerdo con la recomendación del Código Unificado de Buen Gobierno Corporativo de las Sociedades Cotizadas, se observa un notable crecimiento de las consejeras independientes, mientras que las consejeras ejecutivas y las dominicales descienden de forma considerable.

Palabras clave: mujer directiva, IBEX 35, consejera ejecutiva, desigualdad de género, empresas españolas. 


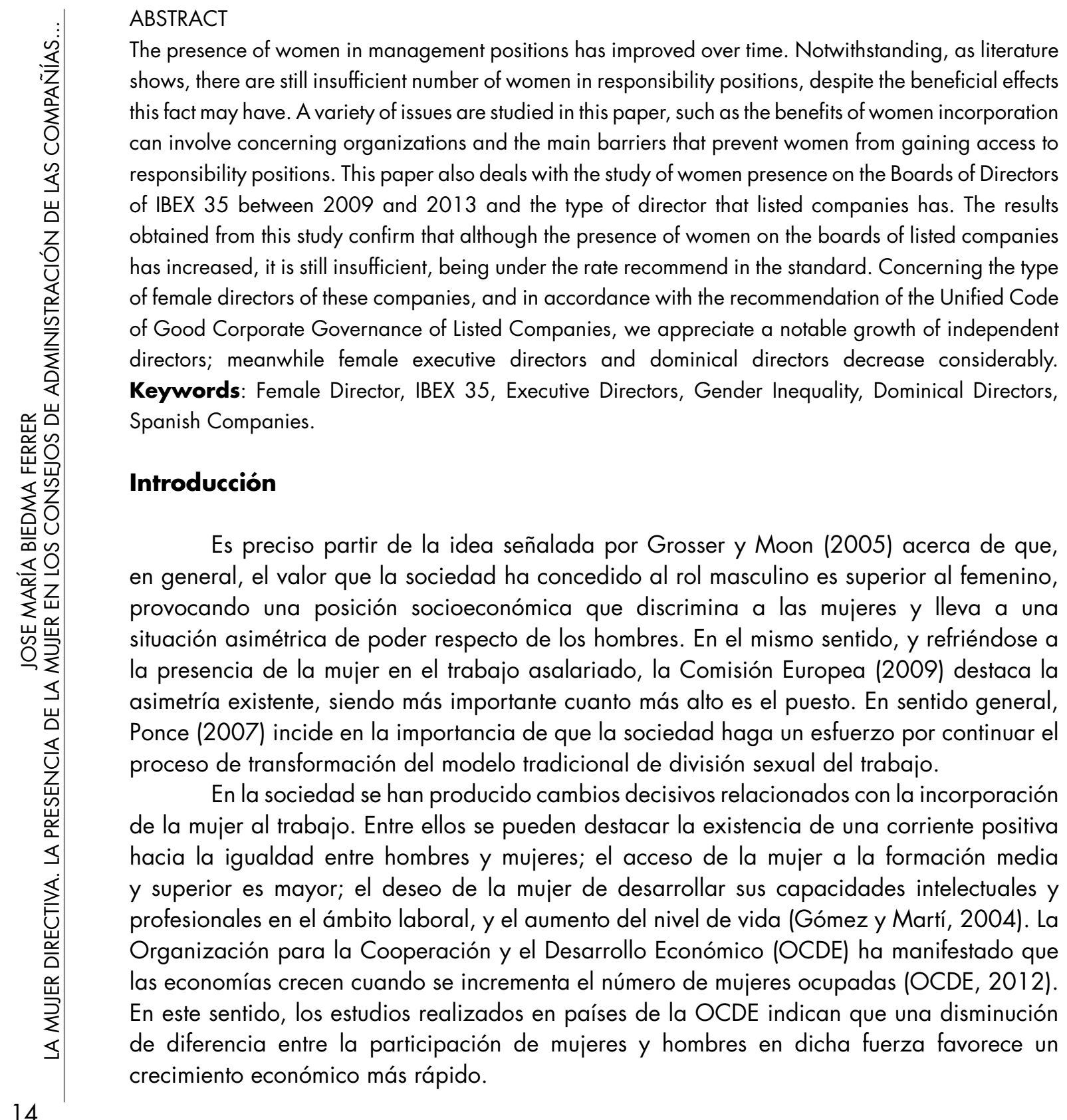

Dossiers Feministes, 22, 2017, 13-27 - ISSN: $1139-1219$ - DOI: http://dx.doi.org/10.6035/Dossiers.2017.22.2 
Si bien el acceso de la mujer al mercado laboral es una realidad, otra cuestión es la presencia de la mujer en puestos directivos. Por ello, este trabajo se plantea dos objetivos: por un lado, realizar una revisión de la literatura sobre la mujer en puestos directivos, los efectos beneficiosos de dicha presencia y las barreras para el acceso a dichos puestos; y, por otro lado, estudiar la evolución de la presencia de la mujer en los consejos de administración de las empresas del IBEX 35, durante el período de 2009-2013, y la tipología de las consejeras de dichas empresas.

La estructura del trabajo se divide en los siguientes apartados: en primer lugar, se realiza una revisión acerca de la mujer en puestos directivos, incluyendo una breve referencia al marco normativo español; posteriormente, se estudian las principales barreras de acceso de la mujer a puestos directivos o de responsabilidad; otro apartado se dedica a la presencia de la mujer en los consejos de las empresas del IBEX 35. Finalmente, se establecen unas conclusiones y recomendaciones.

\section{La mujer en puestos directivos. Breve referencia al marco normativo español}

Como señala Moragas (2012), la mujer actual tiene una buena preparación técnica, pero no está presente de forma adecuada en los órganos directivos de las organizaciones. Con respecto a la preparación técnica, se puede indicar que los datos más recientes publicados por el Ministerio de Cultura, Educación y Deporte (2016), referidos al curso 2015-2016, señalan que el porcentaje de alumnas que supera los estudios universitarios es superior al de alumnos. Concretamente, el $57,31 \%$ de las mujeres frente al $42,69 \%$ de los hombres. Por otra parte, en lo referido a la inadecuada presencia de la mujer en los órganos directivos cabe señalar, siguiendo a Moragas (2012), que ello impide la realización de modificaciones precisas en el funcionamiento de la empresa, con el fin de promover su permanencia y retención en la misma, y al mismo tempo rentabilizar la inversión realizada en ellas.

Siguiendo con la importancia de contar con mayor presencia en las organizaciones, las investigaciones realizadas acerca de las ventajas de la igualdad de oportunidades en el ámbito laboral han señalado beneficios tales como el incremento de la motivación (Chatman et al., 1998) y del nivel de autoestima de los empleados y empleadas (Miner-Rubino et al., 2009). También se ha evidenciado el aumento de la satisfacción laboral (Boada et al., 2005) y del bienestar general (Burke y Mckee, 1996). Finalmente, se observa una disminución del absentismo laboral (Tsui et al., 1992) y de los estereotipos sexistas (Konrad, et al., 1992).

Otra cuestión conectada con el tema que nos ocupa se refiere al hecho de que si se crea un entorno laboral que promueva la diversidad de género, se favorece la incorporación 
a las organizaciones de mujeres altamente cualificadas (Carrasco y Laffarga, 2007). Así, se ha considerado en un estudio realizado (Ramos, 2005) que la incorporación de la mujer a puestos de responsabilidad, además de suponer una cuestión de justicia social e igualdad, es fundamental para las empresas y un valor en alza muy interesante para las organizaciones. Entre los beneficios de la diversidad de personas, cabe destacar el referido a la mejora de la calidad de la resolución de problemas, por cuanto en la literatura se ha evidenciado que la pluralidad de perfiles supone un mayor número y diversidad de soluciones posibles (Barberá et al., 2002; Carrasco y Laffarga, 2007).

Así pues, en la literatura se han estudiado los motivos por los que es positivo el reclutamiento de las mujeres para puestos directivos. Kochan y otros autores (2003) han establecido una serie de motivos que se indican a continuación:

La escasez de talentos fuerza la búsqueda y utilización de las habilidades de todos los empleados, con independencia del género. En este sentido, Jimeno y Redondo (2005) defienden la importancia de que, ante el escenario de creciente competitividad de los mercados, las empresas creen las condiciones para favorecer el desarrollo de las diversas potencialidades ofrecidas por los miembros de la organización (hombres y mujeres). Por ello, entienden que entrar en la batalla de los sexos carece de sentido porque el talento puede estar en cualquier lugar.

Con respecto a las situaciones de crisis económicas, diversos estudios señalan que habitualmente tras las crisis se produce una intensificación del trabajo de las mujeres, especialmente el que no está remunerado (Gálvez y Rodríguez, 2011). Un informe reciente de la Organización Internacional del Trabajo (2015) ha señalado que en el ámbito empresarial se observan prácticas y experiencias que persiguen la atracción y conservación del talento de la mujer, así como el ascenso de la misma a niveles superiores, dentro del escalafón. No obstante, dicho informe considera necesario seguir con acciones de promoción.

La existencia de equipos de trabajo heterogéneos también suele mejorar los resultados de las organizaciones. La sinergia creada entre la perspectiva masculina y la femenina es de gran valor para la elaboración de estrategias empresariales innovadoras y efectivas. En este sentido, Escapa y Martínez (2010) señalan la importancia de compartir en las organizaciones sinergias entre colaboradores y colaboradoras, para lograr objetivos comunes. Al respecto, el estudio realizado por Castaño (2009) señala que es necesario fomentar la participación de las mujeres en los Consejos de Administración por medio de la estructura empresarial española, con el fin de lograr, por un lado, una mayor igualdad de oportunidades y, por otro lado, un incremento de la diversidad en dichos consejos que contribuyan a la mejora del gobierno corporativo. 
Otro motivo señalado por Kochan (2003) es valorizar la capacidad de las mujeres para el conocimiento de las necesidades de potenciales clientes femeninos, porque ello puede facilitar la comprensión y comunicación con ellos.

Pese a los argumentos señalados a favor de una mayor diversidad de género, y si bien es cierto que la ocupación de puestos directivos por las mujeres en las organizaciones se ha incrementado en los últimos años, la presencia femenina en órganos de dirección de empresa aún no es suficientemente representativa. Ello también se ha puesto de manifiesto en el caso de la gran empresa (Charlo y Núñez, 2012). Los resultados del reciente estudio del Grant Thornton International Business Report (2015) referidos a España vienen a corroborar lo anteriormente comentado, porque el porcentaje de cargos directivos ocupados por mujeres en España ha mejorado, pero aún es insuficiente. Concretamente, este informe señala dos cuestiones de interés para este trabajo: por un lado, indica que sólo el $26 \%$ de los cargos directivos están ocupados por mujeres; y, por otro lado, que el $31 \%$ de las empresas españolas no tienen ninguna mujer directiva. Al respecto, se ha señalado en la literatura que aborda dicha temática que es un proceso lento (Castaño et al., 2010; Escribano, 2015) que lleva a mantener una posición pesimista (Castaño et al., 2010).

A nivel mundial, los estudios también confirman la escasa representación femenina como un fenómeno global (Bilimoria, 2000; Tejersen et al., 2009, y Tejersen y Singh, 2008). Así, por ejemplo, en el sector inmobiliario sueco, una investigación reciente corrobora esta infrarrepresentación femenina (Pauli, 2013).

En lo que respecta al marco normativo español, cabe señalar que se han producido modificaciones legislativas encaminadas a lograr la igualdad de género tanto en las estructuras organizativas como en los procedimientos operativos de las empresas. En este sentido, cabe citar la Ley 39/1999 de 5 de noviembre, para Promover la Conciliación de la Vida Laboral y Familiar de las Personas Trabajadoras, y la Ley Orgánica 3/2007 de 22 de marzo para la lgualdad Efectiva entre Mujeres y Hombres (LOIMH).

Es interesante el llamado principio de presencia o composición equilibrada al que hace referencia la exposición de motivos de la citada Ley Orgánica 3/2007. Con este principio se trata de asegurar una representación suficientemente significativa de ambos sexos en órganos y cargos de responsabilidad. De acuerdo con la Disposición adicional primera de dicha Ley, se entiende por composición equilibrada «la presencia de mujeres y hombres de forma que, en el conjunto a que se refiera, las personas de cada sexo no superen el sesenta por ciento ni sean menos del cuarenta por ciento».

Finalmente, cabe citar el Código Unificado de Buen Comportamiento de las Sociedades Cotizadas aprobado en 2006 , donde se reclama la presencia femenina en los consejos. Sin embargo, no establece mínimos al respecto. 


\section{Principales barreras al acceso de la mujer a puestos directivos 0 de responsabilidad}

La escasa presencia femenina en puestos de liderazgo ha atraído la atención de los/as investigadores/as psicosociales, cuyo objetivo fundamental ha sido tratar de identificar los factores que podrían explicar las barreras que dificultan el progreso profesional de las mujeres, en general, y el acceso a los puestos de liderazgo, en particular. En la literatura se han establecido diversas barreras $u$ obstáculos al acceso de la mujer a puestos directivos. En este apartado, de forma aproximativa, se resaltan algunas barreras $u$ obstáculos significativos, de acuerdo con los estudios realizados.

Con respecto al reclutamiento y selección, en el estudio realizado por Molero (2009), se puso de manifiesto que el sexo del evaluador es importante para la valoración de los directivos y directivas. Ello supone que los hombres y las mujeres valoren mejor a los directivos de su mismo sexo. Dado que el número de varones directivos es mayor que el número de mujeres directivas, este aspecto constituye una barrera. En la misma línea se ha manifestado que en el proceso de selección, la igualdad de cualificación, la empresa se inclina por seleccionar a varones, partiendo de la idea de que hombre tiene más necesidad de trabajar que la mujer (Fitzgerald y Weitzman, 1992).

La investigación realizada por Poelmans y otros investigadores (2003) señala que en los procesos de selección la mujer se encuentra discriminada, aunque es preciso ser cauto en las generalizaciones y matizar que esta discriminación depende del tipo de trabajo. Así, por ejemplo, los autores señalan que en el supuesto de que las candidatas sean mujeres jóvenes se produce discriminación a favor del hombre. No obstante, dichos autores matizan que si son trabajos que requieren niveles de formación medio altos y la asunción de responsabilidades es fundamental que la mujer se encuentre soltera. En el caso de que las mujeres jóvenes se encuentren casadas, resultan discriminadas independientemente del tipo de trabajo.

Posteriormente, se ha firmado que, si bien no se observa que las mujeres son discriminadas en el acceso a las entrevistas, los resultados ponen de manifiesto que existen conductas que se relacionan con el llamado fenómeno de segregación ocupacional de género. Ello evidencia que, entre los empleadores, existen visiones estereotipadas sobre la mayor idoneidad para la realización de determinadas tareas (Escot et al., 2008).

En lo referido a los estereotipos de género que producen discriminación, se ha investigado la existencia de creencias que perjudican el acceso de la mujer a puestos directivos. Así, por ejemplo, se ha puesto de manifiesto que existe la creencia de que los rasgos que caracterizan a un buen directivo se corresponden con los propios del hombre 
(Ramos et al., 2002). En la misma línea, se percibe al hombre como empresario exitoso (Hopkins y O’Neil, 2007), no considerando a la mujer adecuada para la asunción de tareas de responsabilidad o liderazgo (Collins, 2005).

También resulta de interés el efecto de las responsabilidades familiares en la mujer para el acceso a puestos de responsabilidad. En este sentido, Albert y otros (2008) plantean la importancia del hecho de la autolimitación profesional de la mujer, con el fin de tratar de lograr la conciliación entre la vida laboral y la familiar. No obstante, todo ello se debe relacionar con la ausencia, en muchas ocasiones, de políticas laborales consolidadas que posibiliten la conciliación del trabajo con la vida familiar (Agut y Martín, 2007).

Otra cuestión es la relacionada con las redes informales y la exclusión de la mujer. Al respecto, cabe señalar que existen diversos estudios que respaldan esta idea (Giosa y Rodríguez, 2010; Albert et al., 2008; Hopkins y O’Neil, 2007).

También ha sido objeto de atención en la literatura la falta de mentoring entre las mujeres profesionales (Bilimoria et al., 2007). Acerca de esta carencia, Medina-Vicent (2015) señala la importancia de promover el mentoring entre las mujeres profesionales como herramienta educativa, con el fin de conseguir la igualdad de género en la alta dirección.

En resumen, la revisión de la literatura realizada permite plantear hasta qué punto es representativa la presencia de la mujer en los consejos de las empresas del IBEX 35 en un determinado período de tiempo. A esta cuestión se dedica el siguiente apartado.

\section{La presencia de la mujer en los consejos de las empresas del IBEX 35}

El IBEX 35 se considera como el índice oficial de la bolsa española. Se compone de 35 empresas, constituyendo un indicador que mide las subidas o bajadas del precio de las acciones. Las empresas que componen este índice se caracterizan porque son las más negociadas. Es decir, aquellas que son más líquidas. Ello significa, entre otras cosas, que no siempre tienen por qué estar las mismas empresas, pero aquellas que forman parte de dicho índice tienen importancia.

En este apartado se estudia la evolución de la presencia de la mujer en los consejos de empresas del IBEX 35 durante el período de 2009-2013. La justificación de la elección de los datos radica en comprobar la evolución de la presencia de la mujer en un período relativamente largo, así como estudiar si el período de crisis ha afectado al número de mujeres consejeras. En la tabla 1 se puede observar, por un lado, el número de empresas cotizadas que contaban con la presencia de alguna mujer en el consejo de administración y, por otro lado, el número total de consejeras del IBEX 35. 
Tabla 1. Presencia de la mujer en los consejos de las empresas del IBEX 35

\begin{tabular}{|c|c|c|c|c|}
\hline $\begin{array}{c}\text { Presencia de } \\
\text { mujeres en los } \\
\text { consejos }\end{array}$ & Sociedades & $\%$ & Consejeras & $\%$ \\
\hline 2004 & 11 & 31,4 & 18 & 3,4 \\
\hline 2005 & 12 & 34,3 & 17 & 3,3 \\
\hline 2006 & 19 & 54,3 & 26 & 5,1 \\
\hline 2007 & 21 & 60 & 30 & 6 \\
\hline 2008 & 26 & 74,3 & 44 & 8,7 \\
\hline 2009 & 27 & 79,4 & 50 & 10,2 \\
\hline 2010 & 29 & 82,9 & 53 & 10,6 \\
\hline 2011 & 32 & 91,4 & 61 & 12,1 \\
\hline 2012 & 31 & 88,6 & 66 & 13,5 \\
\hline 2013 & 32 & 91,4 & 75 & 15,6 \\
\hline & & & & \\
\hline
\end{tabular}

\footnotetext{
Fuente: Elaboración propia a partir de los Informes anuales de Gobierno Corporativo de las compañías del IBEX 35(Años 2004-2013).
}

Del análisis de los datos contenidos en la tablal, se pueden extraer algunas conclusiones de interés para nuestro trabajo:

- Desde 2004 a 2013, salvo en el ejercicio de 2012, se observa un crecimiento del número de empresas que cuentan con alguna mujer en el consejo de administración. Dado que en el año 2013 el 91,4\% de las empresas cotizadas contaba con alguna mujer, es evidente que desde el año 2004 el dato se ha casi triplicado, siendo en este sentido positivo el avance que se ha llevado a cabo.

- En lo que respecta al número de mujeres consejeras desde que se inició la crisis económica, se observa un incremento porcentual significativo cercano al $75 \%$. De ello puede deducirse que la crisis, en términos porcentuales, no ha afectado negativamente a la presencia de mujeres en los consejos de administración de las empresas del IBEX 35. El Informe ADD TALENTIA (2015) recuerda que las mujeres están contribuyendo a salir de la crisis.

- También resulta significativo el incremento del número de mujeres en el período comprendido. Se ha pasado de tener a 18 mujeres en 2004 como consejeras 
de las empresas cotizadas a un total de 75 mujeres en 2013. Ello supone un incremento del $12,2 \%$. A pesar de este incremento, el porcentaje de mujeres consejeras en 2013 se sitúa en el 15,6\%. Pese a todo, el porcentaje está aún lejos del $40 \%$ recomendado.

Todo lo anteriormente señalado sigue constatando la escasa presencia de la mujer en puestos de responsabilidad. Ello conecta con lo señalado por Castaño (2010) cuando afirma que la incorporación de las mujeres a los puestos directivos en las empresas españolas supone un proceso lento que no permite ser demasiado optimistas al respecto.

La recomendación 13 del Código Unificado de Buen Gobierno de las Sociedades Cotizadas (2013), además de recordar la necesidad de alcanzar una adecuada diversidad de género en los Consejos de Administración, llama la atención sobre el hecho de que desaprovechar el talento empresarial de las mujeres, que suponen más del $50 \%$ de la población, no puede ser económicamente racional en el conjunto de las grandes empresas españolas. El citado código invita a que se realice un esfuerzo adicional para que aumente la presencia de la mujer en los puestos de alta dirección y a los Consejos de Administración. Por ello, y como complemento a los requisitos de transparencia señalados en la Orden ECC/461/2013, el Código insta a las sociedades que tienen una escasa presencia femenina en sus Consejos a que se esfuercen de forma deliberada para buscar posibles candidatas, con el fin de cubrir las vacantes que surjan en el Consejo, poniendo especial atención en los puestos de consejeros independientes.

Conviene recordar que el Código Unificado (2006) establece cuatro tipos de consejeros: ejecutivos, dominicales, independientes y otros. En este sentido, tal y como resume Fernández et al. (2009), el primer tipo se refiere a los consejeros con un desempeño en la alta dirección, ya sea en su propia empresa o en la de otro grupo empresarial; el segundo tipo hace referencia a accionistas importantes; el tercer tipo engloba consejeros que no tienen ningún tipo de relación personal o profesional con la sociedad, con directivos o con accionistas significativos, cuya misión es la defensa teórica de los intereses del accionariado minoritario; finalmente, se encuentran los denominados «otros consejeros», que son aquellos que no se encuadran en ninguno de los tipos señalados anteriormente.

Para comprobar en qué grado se ha cumplido la recomendación anteriormente señalada, la tabla 2 establece la tipología de las consejeras en el período estudiado. 
Tabla 2. Tipología de consejeras

\begin{tabular}{|c|c|c|c|c|}
\cline { 2 - 5 } \multicolumn{1}{c|}{} & \multicolumn{3}{c|}{ Tipología de consejeras } \\
\hline $\begin{array}{c}\text { Presencia de mujeres } \\
\text { en los consejos }\end{array}$ & Ejecutiva & Dominical & Independiente & Otras \\
\hline $\mathbf{2 0 0 4}$ & $22,2 \%$ & $50,0 \%$ & $27,8 \%$ & $0,0 \%$ \\
\hline $\mathbf{2 0 0 5}$ & $11,8 \%$ & $47,1 \%$ & $41,2 \%$ & $0,0 \%$ \\
\hline $\mathbf{2 0 0 6}$ & $15,4 \%$ & $42,3 \%$ & $42,3 \%$ & $0,0 \%$ \\
\hline $\mathbf{2 0 0 7}$ & $6,7 \%$ & $36,6 \%$ & $56,7 \%$ & $0,0 \%$ \\
\hline $\mathbf{2 0 0 8}$ & $4,5 \%$ & $29,6 \%$ & $63,6 \%$ & $2,3 \%$ \\
\hline $\mathbf{2 0 0 9}$ & $4,0 \%$ & $32,0 \%$ & $64,0 \%$ & $0,0 \%$ \\
\hline $\mathbf{2 0 1 0}$ & $3,8 \%$ & $26,4 \%$ & $67,9 \%$ & $1,9 \%$ \\
\hline $\mathbf{2 0 1 1}$ & $3,3 \%$ & $27,9 \%$ & $65,6 \%$ & $3,2 \%$ \\
\hline $\mathbf{2 0 1 2}$ & $4,5 \%$ & $25,8 \%$ & $66,7 \%$ & $3,0 \%$ \\
\hline $\mathbf{2 0 1 3}$ & $4,0 \%$ & $22,7 \%$ & $72,0 \%$ & $1,3 \%$ \\
\hline & & & & \\
\hline
\end{tabular}

Fuente: elaboración propia a partir de los Informes anuales de Gobierno Corporativo de las compañías del IBEX 35(Años 2004-2013).

Los datos estudiados apuntan un notable crecimiento de las consejeras independientes, mientras que las consejeras ejecutivas y las dominicales han tenido un descenso notable. En el caso de las consejeras independientes, durante el período estudiado, el incremento ha supuesto más del $150 \%$.

En este caso, se han seguido las recomendaciones del Código Unificado de Buen Gobierno Corporativo de las Sociedades Cotizadas [CUGC] (2006), sancionado por la Comisión Nacional del Mercado de Valores (CNMV). Dicho Código recomienda que el número de consejeros independientes sea el mínimo posible, con el fin de que los consejeros externos dominicales e independientes supongan una amplia mayoría del Consejo. No obstante, como recuerda Mateu de Ros (2007), el mínimo al que se hacía referencia con anterioridad se suaviza al tener en cuenta el grado de complejidad del grupo societario, así como el porcentaje de participación que tengan los consejeros ejecutivos en el capital social. 


\section{Conclusiones y recomendaciones}

La realización del presente trabajo permite extraer las siguientes conclusiones.

El acceso de la mujer al mercado laboral es una realidad incontestable. No obstante, en lo que respecta a la presencia de la mujer en puestos directivos, sigue siendo escasa e insuficiente, pese a haber mejorado.

Para las organizaciones, la existencia de beneficios debido a la presencia de la mujer en puestos de responsabilidad ha sido puesta de manifiesto en diversos estudios (Kochan et al., 2003; Jimeno y Redondo, 2005). Sin embargo, existen todavía diversas barreras que dificultan de forma notable el acceso de las mujeres a puestos directivos (Albert et al., 2008; Molero, 2009; FitzGerald y Weitzman, 1992; Escot et al., 2008).

En el caso de la presencia de la mujer en los consejos de las empresas del IBEX 35, durante el período estudiado (2004-2013), la presencia de las consejeras ha aumentado, pero no de modo suficiente. Sin negar la importancia de estos avances, el porcentaje de presencia de la mujer en puestos de responsabilidad está aún lejos del $40 \%$ recomendado en la normativa española vigente.

Con respecto al número de consejeras independientes de las empresas cotizadas en el IBEX 35, se ha reducido de forma considerable durante el período estudiado, de acuerdo con las indicaciones del Código Unificado de Buen Gobierno Corporativo de las Sociedades Cotizadas (2006).

En este sentido, son múltiples las recomendaciones que se pueden realizar, con el fin de incrementar la presencia de la mujer en puestos de responsabilidad. Una de las más importantes pasaría por concienciar a las organizaciones de los beneficios que puede generar la mujer directiva. La existencia de estudios al respecto (Castaño, 2009; Ramos, 2005; Kochan et al. (2003) avalan esta idea.

También es necesario realizar avances en la legislación que favorezcan la mayor presencia femenina en puestos de responsabilidad. El problema es que, con la legislación vigente, España sólo recomienda, pero no obliga. La elaboración de normas que constriñan a las organizaciones a contar con un porcentaje adecuado de mujeres directivas podría ayudar a paliar la situación existente.

Para futuros trabajos de investigación, se propone continuar estudiando la evolución de la presencia de la mujer en puestos de responsabilidad en empresas que del IBEX 35, con el fin de poder observar las posibles mejoras en este tema. 


\section{Bibliografía}

Agut, Sonia y MARTín, Pilar (2007): «Factores que dificultan el acceso de las mujeres a puestos de responsabilidad: una revisión teórica». Apuntes de Psicología, Vol. 25. № 2, pp. 201-214.

Albert López-lbor, Rocío, Escot, Lorenzo, Fernández, José Andrés y Mateos de Cabo, Ruth (2008): Análisis de la presencia de las mujeres en los puestos directivos de las empresas madrileñas. Colección de Estudios (Consejo Económico y Social), № 29.

BarberÁ, Esther, Ramos, Amparo; Sarrió, Maite, y Candela, Carlos (2002): «Más allá del techo de cristal. Diversidad de género», Revista del Ministerio de Trabajo y Asuntos Sociales. $N^{\circ} 40$, pp. $55-68$.

BILIMORIA, Diana (2000): «Building the business case for women corporate directors», en BURKE, R.J. y MATHIS, M.C. (Eds.): Women on Corporate Boards of Directors: International Challenges and Opportunities, Kluwer Academic Publishers, Dordrecht, pp. 25-40.

BILIMORIA, Diana, GodwIN, Lindsey y ZeleCHOWSKI, Deborah Dahlern (2007): Handbook on women in business and management, New York, Edward Elgar.

Boada i Grau, Joan, Diego, Raúl, Agullo, Esteban y Mañas, Miguel Ángel (2005): «El absentismo laboral como consecuencia de variables organizacionales», Psichotema. Vol. 17, No 2, pp. 212-218.

BURKE, Ronald y MCKEE, Carol (1996): «Do women at the top make a difference? Gender proportions and the experiences of managerial and professional women», Human Relation. Vol. 49, pp. 1093-1104.

Carrasco, Amalia y Lafarga, Joaquina (2.006): «La participación de las mujeres en la toma de decisioneseconómicas en las empresas», Working Paper $n^{\circ} 1$, Proyecto 121/05, Plan Nacional de I+D Instituto de la Mujer.

CASTAÑ̃, Cecilia (2009): Mujeres y poder empresarial en España, Madrid, Ministerio de lgualdad.

Castaño, Cecilia, Martin, Juan, Vázquez, Susana y Martínez, José Luis (2010): «Female executives and the glass ceiling in Spain», International Labour Review. Vol. $149 \mathrm{~N}^{\circ}$ 3, pp. 343-360.

Charlo, María José y NúÑEZ, Miriam (2012): «La mujer directiva en la gran empresa española: perfil, competencias y estilos de dirección», Estudios gerenciales. Vol. $28 \mathrm{~N}^{\circ} 124$, pp. 87-105.

Chatman, Jennifer, Polzer, Jeffrey, Barsade, Sigal y Neale, Margaret (1998): «Being different yet feeling similar: The influence of de demographic composition and organizational culture on work processes and outcomes», Administrative Science Quarterly. № 43, pp. 749-780. 
Código Unificado de buen gobierno de las sociedades cotizadas (2013). Disponible en https:// www.cnmv.es/DocPortal/Publicaciones/CodigoGov/CUBGrefundido_JUNIO2013. pdf [Consultado el 15/05/2017]

ColuINS, Grainne (2005): «The gendered nature of mergers», Gender, Work \& Organization. Vol. $12 \mathrm{~N}^{\circ} 3$, pp. 270-290.

COMISIÓN EUROPEA (2009): Igualdad entre mujeres y hombres. Comunicación de la Comisión al Consejo, al Parlamento Europeo, al Comité Económico y Social Europeo y al Comité de las Regiones, Bruselas, Comisión Europea.

Escapa, Rosa y Martínez, Luz (2010): Estrategias de liderazgo para mujeres directivas, Barcelona, Departament de Treball de la Genralitat de Catalunya.

Escot, Lorenzo, Fernández, José Andrés, Albert lópez-IBOR, Rocío, SAmed, María Obdulia (2008): «Un experimento de campo para analizar la discriminación contra la mujer en los procesos deselección de personal», Cuadernos de Trabajo, 09/2008.

Escribano, $M^{a}$ Cristina (2015): «El acceso de la mujer a los Consejos de Administración de las sociedades mercantiles: igualdad de género y poder de decisión en el Derecho español», en Estudios sobre el futuro Código Mercantil: libro homenaje al profesor Rafael Illescas Ortiz. Getafe. Universidad Carlos III de Madrid, pp. 649-665.

FERNÁNDEZ, Enrique; ARRONDO, Rubén y FernáNDEZ, Carlos (2009): «La retribución de los consejeros en las empresas cotizadas», Boletín Económico de ICE, N N 2961, pp. 35-43.

FitzGerald, Louise F. y Weitzman, Lenore M. (1992): «Women's Career Development: Theory and Practice from a Feminist Perspective», en LEA, H.D. y LeIBOWITZ, Z.B. (Eds): Adult Career Development, Alexandria, The National Career Development Association, pp. 124-160.

Gálvez, Lina y RodRíGuez, Paula. (2011): «La desigualdad de género en las crisis económicas», Investigaciones Feministas, № 2, pp. $113-132$.

Giosa, Noemí y RodríGuez, Corina (2010). Estrategias de desarrollo y equidad de género: una propuesta de abordaje y su aplicación al caso de las industrias manufactureras de exportación en México y Centroamérica, México, Cepal Naciones Unidas.

Gómez López-EgeA, Sandalio y MARTí, Carlos (2004). «La incorporación de la mujer al mercado laboral: implicaciones personales, familiares y profesionales, y medidas estructurales de conciliación trabajo-familia», Documento de Investigación ESE Business School, № 557.

Grant Thornton INTERNATIONAL BUSINESS REPORT (2015). «Mujeres directivas: En el camino hacia la alta dirección». Disponible en http://www.grantthornton.es/archivos/women-inbusiness.pdf [Consultado el 15/05/2017]

GrOSSER, Katey MOON, Jeremy (2005): «Gender mainstreaming and Corporate Social Responsibility: Reporting workplace issues», Journal of Business Ethics, № 62, pp. 327-340. 
HoPKINGS, Margaret y O'NELl, Deborath (2007): «Women and success: Dilemmas and opportunities» en BILMORIA, Diana Y PIDERI, Sandy: Handbook on women in business and management, pp. 132-153. INFORME ADD TALENTIA (2015). Presente y futuro de las 2015 mujeres en los consejos de administración, Madrid Add Telentia. Disponible en http://www.addtalentia.com/images/pdf/ InformeAddTalentia2015.pdf [Consultado el 15/05/2017].

COMISIÓN NACIONAL DEL MeRCADO de VAlORES (2004-2013): Informes anuales de gobierno corporativo de las compañías del lbex 35. Comisión Nacional del Mercado de Valores. Disponible en: https://www.cnmv.es/portal/Publicaciones/PublicacionesGN.aspx?id=22 [Consultado el 15/05/2017].

INSTITUTO PARA LA MUJER Y PARA LA IGUALDAD DE OPORTUNIDADES (2015). Alumnado que terminó estudios universitarios. Disponible en http://www.inmujer.gob.es/MujerCifras/Educacion/ AlumnadoUniversitario.htm [Consultado el 15/05/2017].

JIMENO DE LA MAZA, Francisco Javier y REDONDO, Mercedes (2005): «Sobre el techo de cristal empresarial», AECA: Revista de la Asociación Española de Contabilidad y Administración de Empresas. № 71, pp. 42-46.

Kochan, Thomas, Bezrukova, Katerina, Ely, Robin, Jackson, Susan, Josh Aparna, Jehn, Karen, LEONARD, Jonathan, LEVINE, David y THOMAS, David (2003): «The effects of diversity on business performance: Report of the diversity research network», Human Resource Management, Vol. $42 \mathrm{~N}^{\circ} 1$, pp. 3-21.

KOnRAD, A.; Winter, S. y GUTeK, B. (1992): «Diversity in work group sex composition». Research in the sociology of organizations, Vol. 10, pp. 115-140.

MATEU DE ROS, Rafael (2007). «Los consejeros dominicales y el gobierno de la empresa», Universia Business Review, $4^{\circ}$ trimestre, pp. 10-21.

MEDINA-VICENT, Maria (2015): «Role models, mentoring y redes de mujeres profesionales: educar en valores feministas para fomentar liderazgos éticos», Revista lberoamericana de Producción Académica y Gestión Educativa. №3, pp. 1-19.

Miner-RUbino, Kathi, Settles, Isis y SteWARt, Abigail (2009): «More than numbers: Individual and contextual factors in how gender diversity affects women's», Psychology of women quarterly. Vol. 33, pp. 463-474.

Ministerio de CULTURA, EDUCACIÓN Y DEPORTE (2016): InStITUTO dE LA MUJER PARA LA IGUALDAD dE OPORTUNIDADES. Disponible en: hHp://www.inmujer.gob.es/MujerCifras/Educacion/ AlumnadoUniversitario.htm [Consultada el 28/04/2017].

MOLERO, Fernando (2009): «Mujer y liderazgo en el siglo XXI: una aproximación psicosocial a los factores que dificultan el acceso de la mujer a los puestos de alta responsabilidad. Año 2006 - Año 2009». Estudios e investigaciones, Madrid, Instituto de la Mujer. 
Moragas, María Dolores (2012): «La mujer directiva», Los informes de la CatDem. № 20, pp. 1-12. ORGANIZACIÓN INTERNACIONAL DEL TRABANO (2015): «La mujer en la gestión empresarial. Cobrando impulso. Informe Mundial». Disponible en http://www.ilo.org/wcmsp5/groups/ public/--dgreports/--dcomm/--publ/documents/publication/wcms_335674.pdf. [Consultado el 02/05/2017]

Organización para la cooperación y el desarrollo económico (OCDE) (2012): Gender Equality in Education, Employment and Entrepreneurship: Final Report to the MCM 2012. Paris, OECD. Disponible en http://www.oecd.org/employment/50423364.pdf [Consultado el 22/04/2017]

PaUL, Karin Maria Staffansson (2013): «All of my bosses have been men-on gender structures in the real-estate industry», Property Management. Vol. 31 , N ${ }^{\circ}$. 5, pp. 420-434.

Poelmans, Steven, Chichilla, Nuria y Martí, Ignasi (2003): Criterios de decisión en los procesos de selección en España ¿̇e discrimina a la mujer?, Navarra, Fundación ADECCO-

PONCE, José Miguel (2007): «La conciliación entre la vida personal y la profesional: un reto para las empresas y sus empleados», Revista Empresa y Humanismo. Vol. 10, № 1, pp. 181-208.

Ramos, Amparo (2005): «Mujeres directivas: un valor en alza para las organizaciones laborales», Cuadernos de Geografía. № 78, pp.191-214.

Ramos, Amparo, Sarrió, Maite, BarberÁ, Ester, Candela, Carlos (2002): «El techo de cristal en la promoción profesional de las mujeres». Revista de Psicología Social. Vol. $17 \mathrm{~N}^{\circ}$ 2, pp. 167-182.

TERJESEN, Siriy y SINGH, Val (2008): «Female presence on corporate boards: a multi country of environmental context», Journal of Business Ethics, Vol. 83 №. 1, pp. 55-63.

TerJeSEN, Siri, Seal, Ruth y SingH, Val (2009): «Women directors on corporate boards: a review and research agenda», Corporate Governance: An International Review. Vol. $17 \mathrm{~N}^{\circ}$. 3, pp. 320-337.

Tsul, Anne, Egan, Terry y O'ReAlly, Charles (1992): «Being different: Relational demography and organizational attachment», Administrative Science Quarterly. Vol. 37, pp. 549-579.

Recibido el 26 de febrero de 2017

Aceptado el 9 de mayo de 2017

BIBLID [1 139-1219 (2017) 22: 13-27] 